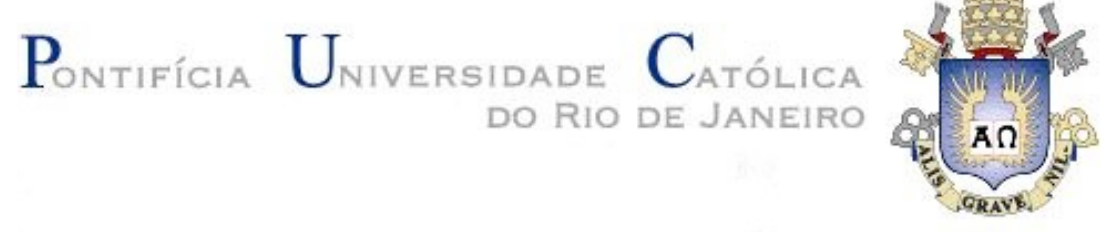

Fernando Hey

Estratégias de Controle Aplicadas ao Problema de Dirigibilidade de Veículos Terrestres em Trajetórias

Fechadas Pré-Definidas

Dissertação de Mestrado

Dissertação apresentada como requisito parcial para obtenção do título de Mestre pelo Programa de PósGraduação em Engenharia Mecânica da PUC-Rio.

Orientadores: Marco Antonio Meggiolaro Mauro Speranza Neto 
Fernando Hey

Estratégias de Controle Aplicadas ao Problema de Dirigibilidade de Veículos Terrestres em Trajetórias

Fechadas Pré-Definidas

Dissertação apresentada como requisito parcial para obtenção do título de Mestre pelo Programa de PósGraduação em Engenharia Mecânica da PUC-Rio. Aprovada pela Comissão Examinadora abaixo assinada.

Marco Antonio Meggiolaro

Orientador

DEM/PUC-Rio

Mauro Speranza Neto

Co-Orientador

DEM/PUC-Rio

Fernando Ribeiro da Silva

DEM/IME

Ricardo Tanscheit

DEE/PUC-Rio

Alexandre de Lima Spinola

PUC-Rio

Prof. José Eugenio Leal

Coordenador Setorial do Centro

Técnico Científico - PUC-Rio

Rio de Janeiro, 14 de março de 2008 
Todos os direitos reservados. É proibida a reprodução total ou parcial do trabalho sem autorização da universidade, do autor e do orientador.

\section{Fernando Hey}

Engenheiro de Controle e Automação formado pela PUCRio em 2005, ingressou para o mestrado, no departamento de engenharia mecânica da própria PUC em 2005. Durante a graduação estagiou na área de engenharia, no desenvolvimento de sistemas integrados de gestão envolvendo softwares de análise estrutural e estabilidade para a industria offshore. Depois de formado trabalha no ramo offshore, na área de operações e instalações submarinas.

Ficha Catalográfica

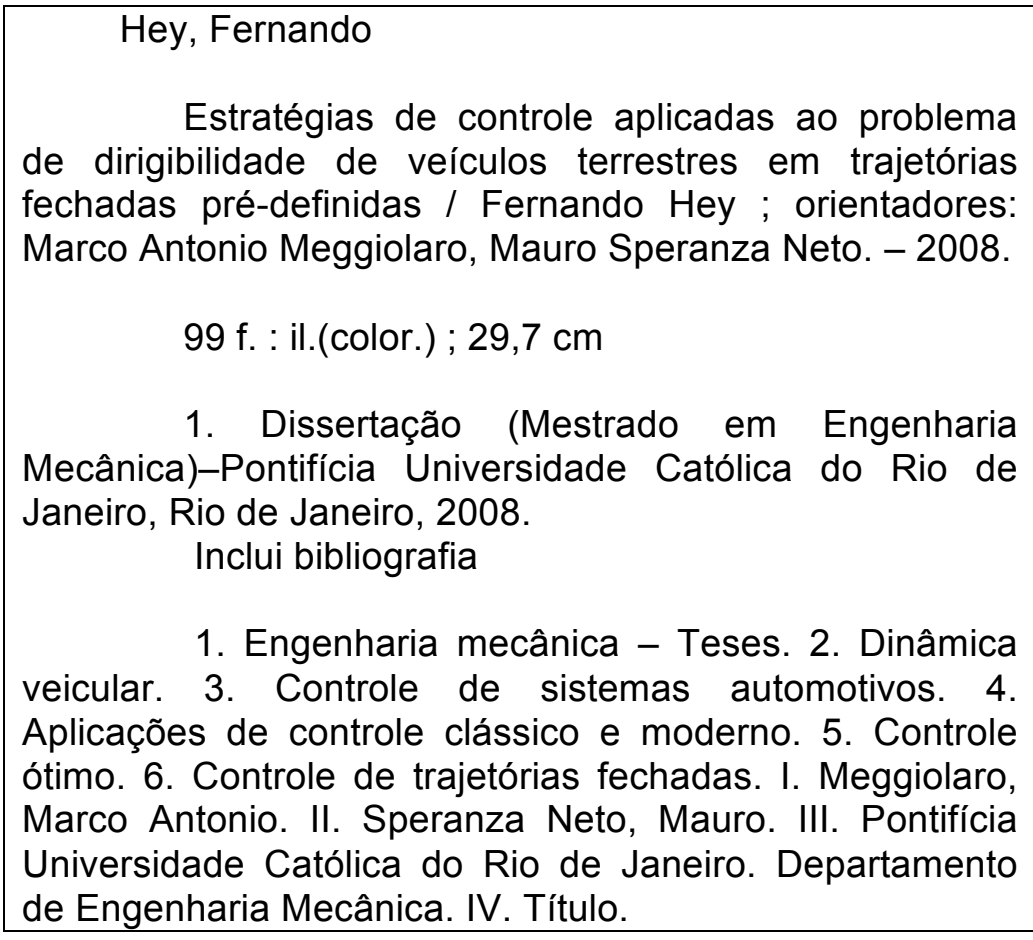

CDD: 621 


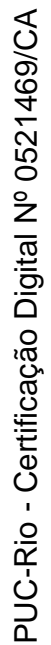

Aos meus familiares e em especial à minha namorada Carolina. 


\section{Agradecimentos}

Ao meu orientador Professor Mauro Speranza pelo estimulo e orientação na realização desse trabalho.

À minha namorada Carolina Caldas pelo grande incentivo e companheirismo nos momentos decisivos do trabalho.

Aos meus amigos Mauro Lawall e Pedro Drummond pela ajuda e pelos momentos de descontração.

Aos meus pais pela educação e conselhos valiosos.

À CAPES pelo incentivo e credibilidade deposita nessa trabalho. 


\section{Resumo}

Hey, Fernando; Speranza Neto, Mauro. Estratégias de Controle Aplicadas ao Problema de Dirigibilidade de Veículos Terrestres em Trajetórias Fechadas Pré-Definidas. Rio de Janeiro, 2008. 99p. Dissertação de Mestrado - Departamento de Engenharia Mecânica, Pontifícia Universidade Católica do Rio de Janeiro.

Apresenta-se o uso das ferramentas lineares de Controle Clássico (Lugar Geométrico das Raízes) e Moderno (Realimentação de Estado e de Saída e Alocação de Pólos) para estabelecer os ajustes dos controladores adotados no problema de acompanhamento de trajetórias em traçados fechados por veículos terrestres, procurando reproduzir o comportamento do ser humano no comando deste tipo de sistema. Os modelos adotados para o veículo são lineares (funções de transferência e matrizes de estado e de entrada), porém a caracterização da trajetória fechada é geometricamente não-linear. Verifica-se deste modo como o projeto de um controle linear satisfaz as condições não lineares associadas. Os conceitos e ferramentas conhecidos são aplicados em diversos tipo de traçados, para diferentes condições do veículo - velocidade, limites de esterçamento, etc e, a partir das simulações realizadas, são analisadas as características de comportamento do veículo - acelerações, estabilidade, etc - e comparadas as previsões dos projetos lineares com os resultados encontrados. É feita ainda uma breve introdução ao emprego do Controle Ótimo no problema de acompanhamento de traçados, utilizando um modelo bastante simplificado do veículo, e verificando as condições necessárias para se estabelecer a trajetória ótima em um traçado aberto, dado como critério o tempo mínimo para percorrê-lo.

\section{Palavras-chave}

Dinâmica Veicular; Controle de Sistemas Automotivos; Aplicações de Controle Clássico e Moderno; Controle Ótimo; Controle em Trajetórias Fechadas. 


\section{Abstract}

Hey, Fernando; Speranza Neto, Mauro (Advisor). Control Strategies Applied to Ground Vehicles Handling Problem in Pre-Defined Closed Trajectories. Rio de Janeiro, 2007. 99p. MSc. Dissertation Departamento de Engenharia Mecânica, Pontifícia Universidade Católica do Rio de Janeiro.

The use of classic and modern linear control tools (root locus and output regulation) is presented to determine the parameters of controllers used to follow a pre-defined closed path, in a way to approach the vehicle behavior and human actions when driving a car. The car is represented by linear models (transfer functions, state-space matrix), but the relation between the car and the closed path is non linear. It is verified how the project of a linear controller deals with the non linear characteristics of the closed loop. The concepts and tools of linear control are applied to some kinds of paths in different vehicle conditions (speed, steering angle limits, etc), and the results of simulations show the characteristics of the car, like accelerations, stability and position on the track. It's also presented a little introduction to the problem of determine an optimal trajectory to run a corner, given the initial and final velocities and initial and final positions. In this case a very simple model is considered and the solution is based on open paths analysis.

\section{Keywords}

Vehicular dynamics; Control of automotive systems; Applications of classic and modern control; Optimal control; Closed path control. 


\section{Sumário}

1. Introdução 10

1.1. Motivação 12

1.2. Objetivo Geral 13

1.3. Objetivos Específicos 14

1.4. Revisão Bibliografica 15

$\begin{array}{ll}\text { 1.5. Estrutura da Dissertação } & 15\end{array}$

$\begin{array}{lr}\text { 2. Malha de Controle } & 18\end{array}$

2.1. Construção da Pista 19

2.2. Implementação da Malha de Controle 24

2.3. Geração do Sinal de Erro 26

3. Modelo Cinemático do Veículo 29

3.1. Construção da Malha de Controle 32

3.2. Simulações 33

4. Modelo Linear de Quatro Graus de Liberdade 39

4.1. Malha de Controle com Transferência de Referencial Linear $\quad 40$

4.1.1. Função de Transferência do Modelo 41

4.1.2. Construção da Malha 43

4.1.3. Simulações Para um Controlador Proporcional 46

4.1.4. Controlador Proporcional Derivativo Duplo 49

4.2. Transferência de Referencial Não Linear 54

5. Realimentação do Ângulo de Yaw 60

$\begin{array}{ll}\text { 5.1. Simulações } & 61\end{array}$ 
6. Controlador de Estado 69

6.1. Cálculo da Matriz de Ganho (K) 70

6.2. Simulações Para o Controlador de Estado 73

7. Definição da Trajetória via Controle Ótimo 80

7.1. Descrição do Problema de Tempo Mínimo 81

7.1.1. Condições Iniciais e Finais de Velocidade Livres 81

7.1.2. Condições Iniciais e Finais de Velocidade Definidas 88

7.2. Tratar um Problema Real 94

8. Conclusões 96

9. Referencias Bibliográficas 98 